\title{
Abelian Avalanches and Tutte Polynomials
}

\author{
Andrei Gabrielov \\ Department of Geology, Cornell University, Ithaca, NY 14853 \\ e-mail: andrei@geology.cornell.edu \\ and \\ Institute for Theoretical Physics \\ University of California, Santa Barbara, CA 93106
}

Received November 15, 1992

\begin{abstract}
We introduce a class of deterministic lattice models of failure, Abelian avalanche (AA) models, with continuous phase variables, similar to discrete Abelian sandpile (ASP) models. We investigate analytically the structure of the phase space and statistical properties of avalanches in these models. We show that the distributions of avalanches in AA and ASP models with the same redistribution matrix and loading rate are identical. For AA model on a graph, statistics of avalanches is linked to Tutte polynomials associated with this graph and its subgraphs. In general case, statistics of avalanches is linked to an analogue of a Tutte polynomial defined for any symmetric matrix.
\end{abstract}

Introduction. Different cellular automaton models of failure (sand piles, avalanches, forest fires, etc.), starting with Bak, Tang and Wiesenfeld (BTW) [1], were introduced in connection with the concept of self-organized criticality [2]. Traditionally, all of these models are considered on uniform cubic lattices of different dimensions. Recently Dhar [3] suggested a generalization of the BTW model with an arbitrary (modulo some natural sign restrictions) matrix $\Delta$ of redistribution of accumulated particles during an avalanche. An important property of this Abelian sand pile (ASP) model is the presence of an Abelian (commutative) group governing its dynamics. Abelian sandpiles were studied in [4], and one special case is treated in [5]. In a non-dissipative case $\left(\sum_{j} \Delta_{i j}=0\right.$, for all $\left.i\right)$ an avalanche in the ASP model coincides with a chip-firing game on a graph [6] where $\Delta$ is a Laplace matrix of the underlying graph. 
Another class of lattice models of failure, slider block models introduced in [7] and studied in [8], as well as models [9-13] which are equivalent to quasistatic block models, have continuous time and some quantity which accumulates and is redistributed at lattice sites. This quantity is called the slope, height, stress or energy by different authors. In slider block models it corresponds to force [11]. We use the term height as in [3].

We introduce here a class of deterministic lattice models with continuous time and height values at the sites of the lattice, and with an arbitrary redistribution matrix. For a symmetric matrix, these models are equivalent to arbitrarily interconnected slider block systems. One of these models, which in the case of a uniform lattice coincides with models studied in [10] and in [13] (as series case $a$ ), is characterized by the same Abelian property as ASP models. We call this the Abelian avalanche (AA) model.

The stationary behavior of the AA model is periodic or quasiperiodic, depending on the loading rate vector. We show however that the distribution of avalanches for a discrete, stochastic ASP model is identical to the distribution of avalanches for an arbitrary quasiperiodic trajectory (or to its average over all periodic trajectories) of a continuous, deterministic AA model with the same redistribution matrix and loading rate.

For the AA model on a graph, the combinatorial structure of the phase space and the corresponding statistics of avalanches is described in terms of the invariants of the graph and its subgraphs called "Tutte polynomials" [14]. In the general case, the same is true for an analogue of a Tutte polynomial defined for any symmetric matrix.

In the first section, we introduce different types of avalanche models. In the second section, we investigate the properties of AA models. In the third section, we study the structure of the set of recurrent configurations and derive analytic formulas for the mean number of avalanches in the AA model. Some of our results are new also for ASP models. In the fourth section, we establish the equivalence of distributions of avalanches for AA and ASP models. In the fifth section, we describe the structure of the phase space for AA models on a graph in terms of Tutte polynomials. In the sixth section, we describe the 
distribution of avalanches in the AA model in terms of an analogue of a Tutte polynomial for an arbitrary symmetric matrix. The proofs of the different statements are given in the Appendix.

1. Avalanche models. Let $V$ be a finite set of $N$ elements (sites), and let $\Delta$ be a $N \times N$ real matrix with indices in $V$, with the following properties:

$$
\begin{gathered}
\Delta_{i i}>0, \text { for all } i ; \Delta_{i j} \leq 0, \text { for all } i \neq j ; \\
s_{i}=\sum_{j} \Delta_{i j} \geq 0, \text { for all } i .
\end{gathered}
$$

The value $s_{i}$ is called the dissipation at a site $i$.

At every site $i$, we define a positive real value $h_{i}$ (height). The set $\mathbf{h}=\left\{h_{i}\right\}$ is called the configuration of the system. For every site $i$, a threshold $H_{i}$ is defined, and configurations with $h_{i}<H_{i}$ are called stable. For every stable configuration, the height $h_{i}$ increases in time with a constant rate $v_{i} \geq 0$ until it exceeds a threshold $H_{i}$ at a site $i$. Then the site $i$ breaks, and the heights are redistributed as follows.

$$
h_{j} \rightarrow h_{j}-\Delta_{i j}, \text { for all } j \text {. }
$$

If after this redistribution, any heights exceed thresholds at some other sites, these sites also break according to (3), and so on, until we arrive at a stable configuration and the loading resumes. The sequence of breaks is called an avalanche.

The model (3) has the important Abelian property (see below): the stable configuration of the system after an avalanche, and the number of breaks at any site during an avalanche, do not depend on the order of breaks during the avalanche. We call this model an Abelian avalanche (AA) model.

It may happen that an avalanche continues without end. We can avoid this possibility by suggesting that the system is weakly dissipative in the following sense. We require that from every non-dissipative site $i, s_{i}=0$, there exists a path to a dissipative site $j, s_{j}>0$, 
i.e. a sequence $i_{0}, \ldots, i_{m}$ with $i_{0}=i, i_{m}=j$ and $\Delta_{i_{k-1} i_{k}}<0$, for $k=1, \ldots, m$. It is easy to show that in a weakly dissipative system every avalanche is finite.

We suppose also that the system is properly loaded, i.e. for every site $j$, there exists a path from a loaded site $i, v_{i}>0$, to the site $j$. If this is not the case, some parts of the system do not evolve in time. For a properly loaded system, the rate of breaks at every site is positive.

In the case of a symmetric matrix $\Delta$ and $v_{i}=s_{i}$, for all $i$, this model is equivalent to a system of blocks where $i$-th block is connected to $j$-th block by a coil spring of rigidity $\Delta_{i j}$ and to a slab moving with a unit rate by a leaf spring of rigidity $s_{i}$. For every block, a static friction force $H_{i}$ is defined, and a block is allowed to move by one unit of space when the total force $h_{i}$ applied to this block from other blocks and the moving slab, exceeds $H_{i}$. The dissipation property means that the loading rate is positive at least for one block in every connected component of the system.

Remark 1. The previous definition can be also reformulated for the model where

$$
h_{j} \rightarrow h_{j}-\Delta_{i j} h_{i}, \text { for } j \neq i, \text { and } h_{i} \rightarrow 0
$$

at a break of $i$-th site, studied in $[11,12]$ and in $[13]$ as series case $b$. This corresponds to a system of blocks in which every block stops when the total force acting on it vanishes. In this case, in addition to the redistribution rule, the choice of one or several (e.g. all) possible breaks in fast time should be specified.

Finally, we can introduce a system with parallel redistribution by [13] considering continuous fast time $\theta$ and redistribution rules

$$
\frac{\partial h_{j}}{\partial \theta} \rightarrow \frac{\partial h_{j}}{\partial \theta}-\Delta_{i j} \text {, for all } j,
$$

when the $i$-th element breaks at $h_{i}=H_{i}$, and

$$
\frac{\partial h_{j}}{\partial \theta} \rightarrow \frac{\partial h_{j}}{\partial \theta}+\Delta_{i j}, \text { for all } j,
$$


when the $i$-th element heals at $h_{i}=0$. This corresponds to a system of blocks where several blocks are allowed to slip simultaneously during an avalanche.

These two models, i.e. specified by (4) and by (5) and (6), are not Abelian.

Remark 2. All the models introduced here are deterministic. If we replace uniform loading in time by random loading then a class of stochastic models can be defined. Many of the properties of the deterministic AA model are valid also for the stochastic case.

2. Abelian avalanches. We want to establish the Abelian properties of the model (3). Many of our arguments are similar to those in [3].

The dynamics of the model does not change if we replace the values $H_{i}$ by some other values, and add the difference to all configuration vectors. For convenience we take $H_{i}=\Delta_{i i}$. In this case, $h_{i} \geq 0$ for any trajectory of the system when the $i$-th element has been broken at least once. Hence only configurations with non-negative heights at all sites are relevant for the long-term dynamics. Let $\mathcal{S}=\left\{0 \leq h_{i}<\Delta_{i i}\right\}$ be the set of all stable configurations in $\mathbf{R}_{+}^{V}=\left\{h_{i} \geq 0\right.$, for all $\left.i\right\}$.

Let $\mathbf{h}(t)$ be a trajectory of the model (3), and let $\mathbf{n}=\left\{n_{i}(t), i \in V\right\}$ be the number of breaks of a site $i$ during a time interval $t$. It is easy to show (see Appendix A) that the average rate of breaks per unit time $\mathbf{r}=\mathbf{n}(t) / t$ satisfies

$$
\Delta^{\prime} \mathbf{r} \rightarrow \mathbf{v}, \text { for } t \rightarrow \infty
$$

Here $\Delta^{\prime}$ is transpose of $\Delta$, and $\mathbf{v}$ is the loading rate in a deterministic model, or the mean loading rate in a stochastic model.

As the rate of breaks at every site is positive for a properly loaded system, this implies that $\Delta^{\prime}\left(\mathbf{R}_{+}^{V}\right) \supset \mathbf{R}_{+}^{V}$. In particular, $\Delta$ is nonsingular. He have also $\operatorname{det}(\Delta)>0$ because the set of all weakly dissipative matrices satisfying (1) and (2) is a convex domain containing a unit matrix.

Let $\mathbf{h}$ be any configuration in $\mathbf{R}_{+}^{V}$. Let $i_{1}, \ldots, i_{m}$ be an avalanche started at $\mathbf{h}$, i.e. a sequence of consecutive breaks (3) such that configurations after all breaks but the last 
are unstable and the configuration $\mathbf{h}^{\prime} \in \mathcal{S}$ after the $m$-th break is stable.

It can be shown (see Appendix B) that $\mathbf{h}^{\prime}=\mathcal{A} \mathbf{h}$ does not depend on the possible choice of breaks, and is completely determined by the initial configuration $\mathbf{h}$. More precisely, let $n_{i}$ be the number of times the site $i$ breaks during the avalanche. Then

$$
n_{i} \text { depends only on } \mathbf{h} \text {, for all } i \text {. }
$$

Hence an avalanche operator

$$
\mathcal{A}: \mathbf{R}_{+}^{V} \rightarrow \mathcal{S}
$$

is defined.

For any vector $\mathbf{u} \in \mathbf{R}_{+}^{V}$ we define a loading operator $\mathcal{B}_{\mathbf{u}} \mathbf{h}=\mathbf{h}+\mathbf{u}$. We call $\mathcal{C}_{\mathbf{u}}=\mathcal{A}^{\circ} \mathcal{B}_{\mathbf{u}}$ a load-avalanche operator.

We claim that every pair of load-avalanche operators commute. More precisely, for any $\mathbf{u} \in \mathbf{R}_{+}^{V}$ and $\mathbf{v} \in \mathbf{R}_{+}^{V}$, we have

$$
\mathcal{C}_{\mathbf{u}} \circ \mathcal{C}_{\mathbf{v}}=\mathcal{C}_{\mathbf{u}+\mathbf{v}}
$$

The proof (see Appendix B) follows arguments of [6] for chip-firing games.

Following [3], we define recurrent configurations of AA model as those stable configurations that can be reached after arbitrary long time intervals.

We claim that for a weakly dissipative, properly loaded system the set $\mathcal{R}$ of all of these configurations does not depend on $\mathbf{v}$ and has volume $\operatorname{det}(\Delta)$.

Let $\delta_{i}=\left(\Delta_{i 1}, \ldots, \Delta_{i N}\right)$ be the $i$-th row vector of the matrix $\Delta$. Integer combinations of vectors $\delta_{i}$ generate a lattice $\mathcal{L}$ in $\mathbf{R}^{V}$. Two configurations $\mathbf{h}$ and $\mathbf{h}^{\prime}$, are called equivalent if $\mathbf{h}^{\prime}-\mathbf{h}$ belongs to $\mathcal{L}$. A subset in $\mathbf{R}^{V}$ is called a fundamental domain for $\mathcal{L}$ if, for every configuration $\mathbf{h} \in \mathbf{R}^{V}$, it contains exactly one configuration equivalent to $\mathbf{h}$. The volume of every fundamental domain is equal to $\operatorname{det}(\Delta)$.

The rule (3) for breaks can be rewritten as $\mathbf{h} \rightarrow \mathbf{h}-\delta_{i}$. For every $\mathbf{h} \in \mathbf{R}_{+}^{V}$, configuration $\mathcal{A} \mathbf{h}$ is equivalent to $\mathbf{h}$ and belongs to $\mathcal{S}$. Hence $\mathcal{S}$ contains a fundamental domain for $\mathcal{L}$. 
Let $\mathbf{u} \in \mathbf{R}_{+}^{V}$ and $\mathcal{S}_{\mathbf{u}}=\mathcal{S}+\mathbf{u}$. Then $\mathcal{S}_{\mathbf{u}}$ contains a fundamental domain for $\mathcal{L}$ because this property is translation-invariant. Hence $\mathcal{A}\left(\mathcal{S}_{\mathbf{u}}\right)=\mathcal{C}_{\mathbf{u}}(\mathcal{S})$ contains a fundamental domain for $\mathcal{L}$.

It can be shown (see Appendix C) that

$$
\mathcal{C}_{\mathbf{u}}(\mathcal{S}) \text { is a fundamental domain for } \mathcal{L} \text { if } u_{i} \geq \Delta_{i i} \text {, for all } i \text {. }
$$

The Abelian property (10) implies that the intersection of images of any two loadavalanche operators $\mathcal{C}_{\mathbf{u}}$ and $\mathcal{C}_{\mathbf{v}}$ contains a fundamental domain for $\mathcal{L}$, because it contains $\mathcal{C}_{\mathbf{u}+\mathbf{v}}(\mathcal{S})$, hence the two images coincide when both vectors $\mathbf{u}$ and $\mathbf{v}$ have large enough components.

This proves that $\mathcal{R}$ is a fundamental domain for $\mathcal{L}$, hence its volume is $\operatorname{det}(\Delta)$, when all components of the loading rate vector $\mathbf{v}$ are positive, because all components of $\mathbf{v} t$ are large enough for large values of $t$. If some of $v_{i}$ are 0 , the proper loading condition guarantees that, for large $t$, there exists an avalanche starting at $\mathbf{v} t$ and passing through a vector with large enough components- hence $\mathcal{R}$ is a fundamental domain for $\mathcal{L}$ also in this case.

The dynamics of the system on $\mathcal{R}$ in a deterministic model is defined by the break rate vector $\mathbf{r}=\Delta^{\prime-1} \mathbf{v}$. If this vector is collinear to an integer vector, $T \mathbf{r}=\mathbf{n}$, then $v T=\Delta^{\prime} \mathbf{n}$, hence every trajectory is periodic, with a period $T$, and a site $i$ breaks $n_{i}$ times during a period $T$, for every periodic trajectory. Otherwise, every trajectory is quasiperiodic.

In any case, the measure $d \mathbf{h}=\prod_{i \in V} d h_{i}$ on $\mathcal{R}$ is invariant under the dynamics of the system. This is also true for the random loading.

As a result, (7) has the following implication.

$$
\frac{1}{\operatorname{det}(\Delta)} \int_{\mathcal{R}} \mathbf{n}(\mathbf{h}+\mathbf{v}) d \mathbf{h}=\Delta^{\prime-1} \mathbf{v}
$$

where $\mathbf{n}(\mathbf{h})=\left\{n_{j}(\mathbf{h})\right\}$, and $n_{j}(\mathbf{h})$ is the number of breaks at a site $j$ during an avalanche started at $\mathbf{h}$. 
Let

$$
\mathcal{R}_{i}=\overline{\mathcal{R}} \cap\left\{h_{i}=\Delta_{i i}\right\}
$$

be the set of (unstable) configurations where the recurrent avalanches with a first break at $i$ start. Here $\overline{\mathcal{R}}$ is the closure of $\mathcal{R}$. For any quasiperiodic trajectory of the system (in the periodic case, for a randomly chosen periodic trajectory), the mean (per unit time) number of times it crosses a domain $D \subset \mathcal{R}_{i}$ is equal to

$$
p_{i}(D)=v_{i} \operatorname{Vol}(D) / \operatorname{Vol}(\mathcal{R})=v_{i} \operatorname{Vol}(D) / \operatorname{det}(\Delta)
$$

Here $\operatorname{Vol}(D)$ is the volume in $(|V|-1)$-dimensional space $\left\{h_{i}=\Delta_{i i}\right\}$.

In particular, the mean number of avalanches started at $i$ is equal to

$$
p_{i}\left(\mathcal{R}_{i}\right)=v_{i} \operatorname{Vol}\left(\mathcal{R}_{i}\right) / \operatorname{det}(\Delta)
$$

The mean number of breaks at a site $j$ per unit time can be computed from (14) as

$$
r_{j}=\sum_{i} \frac{v_{i}}{\operatorname{det}(\Delta)} \int_{\mathcal{R}_{i}} n_{j}(\mathbf{h}) d \mathbf{h}_{i}^{\prime}
$$

where $d \mathbf{h}_{i}^{\prime}=d \mathbf{h} / d h_{i}$ is the measure on $\mathcal{R}_{i}$ and $n_{j}(\mathbf{h})$ is the number of breaks at a site $j$ during an avalanche started at $\mathbf{h}$.

Due to $(7), \mathbf{r}=\Delta^{\prime-1} \mathbf{v}$. Hence

$$
\int_{\mathcal{R}_{i}} n_{j}(\mathbf{h}) d \mathbf{h}_{i}^{\prime}=\operatorname{det}(\Delta)\left(\Delta^{-1}\right)_{i j}
$$

and the mean (per avalanche) number of breaks at a site $j$ during avalanches started at a site $i$ is

$$
m_{i j}=\frac{v_{i}}{\operatorname{det}(\Delta)} \int_{\mathcal{R}_{i}} n_{j}(\mathbf{h}) d \mathbf{h}_{i}^{\prime}=\frac{\operatorname{det}(\Delta)\left(\Delta^{-1}\right)_{i j}}{\operatorname{Vol}\left(\mathcal{R}_{i}\right)} .
$$

The value of $\operatorname{Vol}\left(\mathcal{R}_{i}\right)$ is found in the next section, for the case when every site breaks at most once during an avalanche. 
Remark. In the periodic case, a single trajectory can contain avalanches of different sizes, and for a large system, in a time interval shorter than its period, it can be indistinguishable from a chaotic trajectory. This effect (called "periodic chaos") was found in [13] for a uniform lattice.

3. Recurrent configurations. To investigate the structure of the set $\mathcal{R}$ of recurrent configurations, we note first that, for any $\mathbf{h} \in \mathcal{R}$ and any vector $\mathbf{u} \in \mathbf{R}_{+}^{V}$, configuration $\mathbf{h}+\mathbf{u}$ belongs to $\mathcal{R}$ if it belongs to $\mathcal{S}$.

Let $\mathbf{Q}=\left(\Delta_{11}, \ldots, \Delta_{N N}\right)$. For an integer vector $\mathbf{n}$, let $\mathbf{P}=\mathbf{Q}_{\mathbf{n}}=\mathbf{Q}-\Delta^{\prime} \mathbf{n}$ be a configuration equivalent to $\mathbf{Q}$, and let $\mathcal{V}_{\mathbf{n}}=\left\{h_{i}<P_{i}\right\}$ be an open negative octant with a vertex at $\mathbf{P}$. It can be shown (see Appendix D) that

$$
\mathcal{R}=\mathcal{S} \backslash \cup^{\prime} \mathcal{V}_{n}
$$

where the union $U^{\prime}$ is taken over all $\mathbf{n}$ with at least one positive component. If $0 \leq n_{i} \leq 1$, for all $i$, the sets $\mathcal{S} \cap \mathcal{V}_{\mathbf{n}}$ coincide with stable forbidden subconfigurations [3]

$$
\mathcal{F}_{X}=\left\{\mathbf{h} \in \mathcal{S}, h_{j}<-\sum_{i \in X, i \neq j} \Delta_{i j}, \text { for } j \in X\right\} .
$$

Here $X$ is the set of sites $i$ with $n_{i}=1$. Dhar [3] argues that the union of sets (20) over all nonempty subsets of $V$ coincides with $\mathcal{S} \backslash \mathcal{R}$.

In general, this is not true. For a $2 \times 2$ matrix $\Delta$ with $\delta_{1}=(2,-1)$ and $\delta_{2}=(-3,4)$ we have $\mathbf{Q}_{(2,1)}=(1,2)$. Hence configurations with $h_{1}<1$ and $h_{2}<2$ are not recurrent, and only configurations with $h_{2}<1$ are forbidden.

It can be shown, however (see Appendix E) that

$$
\mathcal{R}=\mathcal{S} \backslash \bigcup_{X \subset V} \mathcal{F}_{X},
$$

i.e. all allowed stable configurations (i.e. those that do not contain any forbidden subconfigurations) are recurrent, when

$$
\sum_{i \in V} \Delta_{i j} \geq 0, \text { for all } j .
$$


In particular, this is true when $\Delta$ is symmetric.

Suppose now that

$$
\sum_{i \in V} \Delta_{i j}>0, \text { for all } j
$$

In this case, the configuration $\mathbf{Q}-\sum_{i \in V} \delta_{i}$ is stable. Hence every site can break at most once in an avalanche started at any configuration $\mathbf{h}$ with $h_{i} \leq \Delta_{i i}$, for all $i$.

Let $\mathcal{R}_{i}$ be the set (13) of recurrent configurations initiating avalanches with a first break at $i$. If (23) holds, the values of $\mathbf{h}^{\prime}=\left\{h_{j}, j \in V, j \neq i\right\}$ in $\mathcal{R}_{i}$ are defined, due to (21), by the same inequalities as the set of all allowed configurations for a model (3) on $V \backslash\{i\}$ with a matrix $\Delta(i)$, where $\Delta(i)$ is $\Delta$ with $i$-th row and $i$-th column deleted. Hence $\mathcal{R}_{i}$ coincides with the set of all recurrent configurations for $\Delta(i)$, and

$$
\operatorname{Vol}\left(\mathcal{R}_{i}\right)=\operatorname{det}(\Delta(i))=\operatorname{det}(\Delta)\left(\Delta^{-1}\right)_{i i}
$$

Due to (15), the mean number of avalanches started at a site $i$ per unit time is

$$
p_{i}\left(\mathcal{R}_{i}\right)=v_{i} \operatorname{det}(\Delta(i)) / \operatorname{det}(\Delta)=v_{i}\left(\Delta^{-1}\right)_{i i}
$$

Hence the mean number of avalanches in the system per unit time is equal to

$$
\sum_{i} v_{i}\left(\Delta^{-1}\right)_{i i}
$$

Due to (18), we have

$$
m_{i j}=\left(\Delta^{-1}\right)_{i j} /\left(\Delta^{-1}\right)_{i i}
$$

where $m_{i j}$ is the mean (per avalanche) number of breaks at a site $j$ during avalanches started at a site $i$.

Remark. In case (22) holds but (23) is not valid, the volume of $\mathcal{R}_{j}$ is less than $\operatorname{det}(\Delta(j))$. Due to $(21)$, for every subset $F \subset V \backslash\{j\}$ such that $\Delta_{j j}+\sum_{i \in F} \Delta_{i j}=0$, configurations with $h_{j}=\Delta_{j j}$ and $h_{\nu}<-\Delta_{j \nu}-\sum_{i \in F} \Delta_{i \nu}$, for $\nu \in F$, do not belong to 
$\mathcal{R}_{j}$. It can be shown, however, that the formulas (25)-(27) are still valid for the following modification of the model.

We allow every site to break at most once in an avalanche. At the end of an avalanche started at a site $i$, the value $h_{i}$ can be still at the threshold level $\Delta_{i i}$. In this case we immediately start a new avalanche at a site $i$, and so on until finally we arrive at a stable configuration.

For the original model, (25)-(27) are true if we count every avalanche with the multiplicity of the number of breaks at its starting site.

4. Distributions of avalanches in AA and ASP models. There is obvious similarity between the properties of the deterministic, continuous AA model and the stochastic, discrete ASP model. We want to show that, for a matrix $\Delta$ with integer elements, the distribution of avalanches in the AA model is identical to the distribution of avalanches in the ASP model with the same matrix $\Delta$ and the same loading rate vector $\mathbf{v}$.

For a matrix $\Delta$ with integer elements satisfying (1) and (2), and a loading vector $\mathbf{v}$ with $\sum v_{i}=1$, ASP model is defined as follows. The height $h_{i}$ at every site $i \in V$ is an integer, $0 \leq h_{i}<\Delta_{i i}$. At every (discrete) time step, we choose a site $i$ with a probability $v_{i}$ and add a particle at the site $i$, i.e. add 1 to the height $h_{i}$. If $h_{i}=\Delta_{i i}$ after this operation, we start an avalanche according to the rule (3). After termination of an avalanche, we proceed with adding the next particle. Only uniform loading (all $v_{i}$ equal) was considered in [3]. However, the generalization to any proper loading rate vector is straightforward.

As it is shown in [3], the recurrent configurations for the ASP model are precisely the integer points in the set $\mathcal{R}$ of recurrent configurations of the AA model with the matrix $\Delta$, every point is attended with equal probability, and the total number of these points $\#(\mathcal{R})$ is equal to $\operatorname{det}(\Delta)=\operatorname{Vol}(\mathcal{R})$.

For the ASP model, a recurrent configuration with $h_{i}=\Delta_{i i}$ starting an avalanche at a site $i$ belongs to the set of integer points in the set $\mathcal{R}_{i}$ defined in (13). For a randomly chosen configuration in $\mathcal{R}$, the probability of initiating an avalanche at a site $i$ at any time 
step is equal to $p_{i}=v_{i} \#\left(\mathcal{R}_{i}\right) / \operatorname{det}(\Delta)$. Due to (19), the number of integer points $\#\left(\mathcal{R}_{i}\right)$ in $\mathcal{R}_{i}$ is equal to $\operatorname{Vol}\left(\mathcal{R}_{i}\right)$. Hence $p_{i}=p_{i}\left(\mathcal{R}_{i}\right)$ coincides with the mean number of avalanches initiated at $i$ defined in (15) for AA model. For any integer vector $\mathbf{k}=\left\{k_{j}\right\}$, the set of points $\mathcal{R}_{i, \mathbf{k}} \subset \mathcal{R}_{i}$ where an avalanche with $k_{j}$ breaks at a site $j$, for all $j \in V$, starts coincides with

$$
\left(\mathcal{R}+\sum_{j} k_{j} \delta_{j}\right) \cap \mathcal{R}_{i}
$$

where $\delta_{j}$ is the $j$-th row vector of $\Delta$. Due to (19), the number of integer points in (28) coincides with its volume. Hence the mean number per time step of avalanches started at a site $i$, with $k_{j}$ breaks at a site $j$, which is equal to $v_{i} \#\left(\mathcal{R}_{i, \mathbf{k}}\right) / \operatorname{det}(\Delta)$ for ASP model, coincides with the mean number per unit time of avalanches of the same type for a quasiperiodic trajectory (in the periodic case, for a randomly chosen periodic trajectory) in AA model, which is equal to

$$
p_{i}\left(\mathcal{R}_{i, \mathbf{k}}\right)=v_{i} \operatorname{Vol}\left(\mathcal{R}_{i, \mathbf{k}}\right) / \operatorname{det}(\Delta)
$$

according to (14).

This equivalence implies, in particular, that the size distributions of avalanches in AA and ASP models, with the same matrix $\Delta$ and loading rate $\mathbf{v}$, coincide.

5. Avalanches on graphs. A graph $G$ is a set $V(G)$ of vertices and a set $E(G)$ of edges with a relation or rule of incidence which associates with every edge in $E(G)$ two vertices in $V(G)$ called its ends. An edge with different ends is a link. Its ends are called adjacent vertices, or neighbors. An edge with identical ends is a loop. For every vertex $i$ its degree $d_{i}$ is equal to the number of the edges incident to it, loops counting twice.

If $V^{\prime} \subseteq V(G)$ and $E^{\prime} \subseteq E(G)$ with the ends of any edge in $E^{\prime}$ belonging to $V^{\prime}$ then a graph $H$ with $V(H)=V^{\prime}, \quad E(H)=E^{\prime}$ and the incidence relation induced from $G$ is called a subgraph of $G$. If $V^{\prime}=V$ then $H$ is a spanning subgraph. If $U \subseteq V(G)$ then the subgraph $H=G[U]$ with $V(H)=U$ and $E(H)$ consisting of all edges of $G$ with both ends in $U$ is called an induced subgraph of $G$. 
An $n$-arc is a graph with vertices $i_{1}, \ldots, i_{n}$ and edges $e_{1}, \ldots, e_{n-1}$ where $i_{\nu}$ and $i_{\nu+1}$ are the ends of the edge $e_{\nu}$. A $n$-circuit is a $n$-arc with additional edge $e_{n}$ with ends $i_{n}$ and $i_{1}$. A graph $G$ is connected if any two of its vertices belong to an arc in $G$. A tree is a connected graph without circuits.

The tree number $T(G)$ of a graph $G$ is defined as the total number of different spanning trees of this graph. $T(G)>0$ only for connected graphs, $T(G)=1$ for any tree, and $T(G)=n$ for a circuit of order $n$.

For every edge $e$ in a graph $G$, the operation of deletion $G-e$ is defined by removing $e$ from $E(G)$, and the operation of contraction $G / e$ is defined by removing $e$ and identifying the ends of $e$ in $V(G)$. It is easy to show [14, p.40] that

$$
T(G)=T(G-e)+T(G / e)
$$

for every link $e$ of $G$. The functions with this property are often called Tutte polynomials.

We consider only loopless graphs, with multiple edges, and define $G / e$ as a graph with loops removed after contraction of $e$. The property $(30)$ of $T(G)$ remains valid for this operation. We define the order $|G|=|V(G)|$ of a graph $G$ as the number of its vertices.

The Laplace matrix $\Delta(G)$ of a graph $G$ is defined as $\Delta(G)_{i i}=d_{i}$ and $-\Delta(G)_{i j}$ equal to the number of links between vertices $i$ and $j$, for $i \neq j$. We have $\sum_{j} \Delta(G)_{i j}=0$, for all $i$.

With any diagonal matrix $S$, with non-negative elements $S_{i i}=s_{i}$, we can associate an avalanche model with a symmetric matrix $\Delta_{S}=\Delta(G)+S$. (The models with nonsymmetric matrices can be associated with directed graphs, but we do not consider this here.)

Let $\mathbf{v}=\left\{v_{i}, i \in V(G)\right\}$ be a loading vector for this model. Suppose that $v_{i}=s_{i}$, for all $i$, as in a slider block model. Then every recurrent trajectory of the AA model with matrix $\Delta_{S}$ and loading rate $\mathbf{v}$ is periodic, with a period $T=1$, and every vertex of $G$ breaks once during this period. 
Let $G_{1}, \ldots, G_{m}$ be an arbitrary partition of $G$ into induced subgraphs, and $i_{\nu}$ a selected vertex in $G_{\nu}$. We claim (see Appendix F) that the total volume occupied by recurrent configurations generating periodic trajectories with an ordered sequence of avalanches covering sets $V\left(G_{1}\right), \ldots, V\left(G_{m}\right)$ initiated at sites $i_{1}, \ldots, i_{m}$ is equal to

$$
s_{i_{1}} T\left(G_{1}\right) \cdots s_{i_{m}} T\left(G_{m}\right) / m !
$$

In particular, this volume does not depend on the order of $G_{\nu}$.

Hence the total volume occupied by periodic trajectories with avalanches constituting a partition of $G$ into induced subgraphs $G_{1}, \ldots, G_{m}$ is equal to

$$
S\left(G_{1}\right) T\left(G_{1}\right) \cdots S\left(G_{m}\right) T\left(G_{m}\right)
$$

where $S\left(G_{\nu}\right)=\sum_{i \in V\left(G_{\nu}\right)} s_{i}$.

As the total volume of all recurrent configurations is $\operatorname{det}\left(\Delta_{S}\right)$, we have

$$
\sum_{m \geq 1} \sum_{G_{1}, \ldots, G_{m}} \prod_{\nu=1}^{m} S\left(G_{\nu}\right) T\left(G_{\nu}\right)=\operatorname{det}\left(\Delta_{S}\right)
$$

where the sum is taken over all partitions of $G$ into induced subgraphs.

The linear term in $S$ in this expression appears for $m=1$ and $G_{1}=G$. It is equal to $S(G) T(G)$. Hence

$$
T(G)=\left.\frac{\partial \operatorname{det}\left(\Delta_{S}\right)}{\partial s_{i}}\right|_{S=0}=\operatorname{det}\left(\Delta_{i}(G)\right), \text { for every } i \in V
$$

Here $\Delta_{i}(G)$ is the matrix $\Delta(G)$ with the $i$-th row and column removed. This is the Matrix-Tree Theorem for graphs [14, p.141].

The mean number of avalanches in a randomly chosen periodic trajectory is equal to

$$
\operatorname{det}\left(\Delta_{S}\right)^{-1} \sum_{m \geq 1} m \sum_{G_{1}, \ldots, G_{m}} \prod_{\nu=1}^{m} S\left(G_{\nu}\right) T\left(G_{\nu}\right) .
$$

Comparing expressions (35) and (26) for the mean number of avalanches, we have an identity

$$
\sum_{m \geq 1} m \sum_{G_{1}, \ldots, G_{m}} \prod_{\nu=1}^{m} S\left(G_{\nu}\right) T\left(G_{\nu}\right)=\operatorname{det}\left(\Delta_{S}\right) \sum_{i} s_{i}\left(\Delta_{S}^{-1}\right)_{i i}
$$


where the sum in the left part is taken over all partitions of $G$ into induced subgraphs.

Let $H$ be an induced subgraph of $G$, and let $G \backslash H$ be an induced subgraph of $G$ with $V(G \backslash H)=V(G) \backslash V(H)$.

For $i \in V(H)$, the total volume of all periodic trajectories with an avalanche started at $i$ covering $H$ is equal, due to (31), to

$$
X(i, H)=s_{i} T(H)\left[\sum_{m \geq 1} \sum_{G_{1}, \ldots, G_{m}} \prod_{\nu=1}^{m} S\left(G_{\nu}\right) T\left(G_{\nu}\right)\right],
$$

where the sum is taken over all partitions of $G \backslash H$ into induced subgraphs.

Applying (33) to $G \backslash H$, we have

$$
X(i, H)=s_{i} T(H) \operatorname{det}\left(\Delta_{S}(G \backslash H)\right) .
$$

Here $\Delta_{S}(G \backslash H)$ is the Laplace matrix of $G \backslash H$ with $s_{\nu}$ added to diagonal elements, for $\nu \in V(G) \backslash V(H)$.

We have also $X(i, H)=s_{i} \operatorname{Vol}\left(\mathcal{R}_{i, H}\right)$, where $\mathcal{R}_{i, H}$ is the subset of $\mathcal{R}_{i}$ where the avalanches covering $H$ start. Hence, if $s_{i}>0$ then

$$
\operatorname{Vol}\left(\mathcal{R}_{i, H}\right)=T(H) \operatorname{det}\left(\Delta_{S}(G \backslash H)\right)
$$

Due to (14), the mean (per unit time) number of avalanches started at $i$ covering $H$ is $p_{i}\left(\mathcal{R}_{i, H}\right)=v_{i} \operatorname{Vol}\left(\mathcal{R}_{i, H}\right) / \operatorname{det}\left(\Delta_{S}(G)\right)$, for arbitrary loading rate $\mathbf{v}$.

Hence

$$
p_{i}\left(\mathcal{R}_{i, H}\right)=v_{i} T(H) \operatorname{det}\left(\Delta_{S}(G \backslash H)\right) / \operatorname{det}\left(\Delta_{S}(G)\right)
$$

for arbitrary loading rate vector. Due to section 4, this is also equal to the mean (per one time step) number of avalanches started at $i$ and covering $H$ in ASP model if all $s_{\nu}$ are integer.

Finally, we have the following expression for the mean number of avalanches of size $k$, both in the AA and ASP models.

$$
\frac{1}{\operatorname{det}\left(\Delta_{S}(G)\right)} \sum_{U \subset V(G),|U|=k} T(G[U]) \operatorname{det}\left(\Delta_{S}(G \backslash G[U])\right) \sum_{i \in U} v_{i}
$$


This gives a purely combinatorial expression for the distribution of the avalanches of different sizes. Explicit formulas for this distribution are found in [13] for a circuit of arbitrary order.

Remark. The formulas (40) and (41) are valid when $s_{i}>0$, for all $i$ with $v_{i}>0$ (because (39) is not true when $s_{i}=0$ ). When $s_{i}=0$ and $v_{i}>0$ for some $i$, these formulas are still valid for the modification of the model suggested in remark at the end of section 3 , when every site is allowed to break at most once during an avalanche.

6. Tutte polynomials for matrices. For any symmetric matrix $\Delta$ with indices in a set $V$, we define a symmetric matrix $\Delta^{\prime}=D_{i j}(\Delta)$ (deletion of $\left.(i, j)\right)$ as

$$
\Delta_{i i}^{\prime}=\Delta_{i i}+\Delta_{i j}, \Delta_{j j}^{\prime}=\Delta_{j j}+\Delta_{i j}, \Delta_{i j}^{\prime}=0
$$

with other elements of $\Delta$ unchanged, and a symmetric matrix $\Delta^{\prime \prime}=C_{i j}(\Delta)$ (contraction of $(i, j))$ as

$$
\Delta_{i i}^{\prime \prime}=\Delta_{i i}+\Delta_{j j}+2 \Delta_{i j}, \Delta_{i k}^{\prime \prime}=\Delta_{i k}+\Delta_{j k}, \text { for } k \neq i, j,
$$

with the $j$-th row and column of $\Delta$ removed and other elements of $\Delta$ unchanged.

For $\Delta=\Delta(G)$, the Laplace matrix of a graph $G$, the matrix $D_{i j}(\Delta)$ is the Laplace matrix of $G$ after deletion of all edges connecting $i$ and $j$, and $C_{i j}(\Delta)$ is the Laplace matrix of $G$ after contraction of all edges with ends at $i$ and $j$.

For every symmetric matrix $\Delta$, let $s_{i}=\sum_{j} \Delta_{i j}$, and let $\Delta_{0}$ be the matrix $\Delta$ with diagonal terms $\Delta_{i i}$ replaced by $\Delta_{i i}-s_{i}$, for all $i$. Then

$$
C_{i j}\left(\Delta_{0}\right)=C_{i j}(\Delta)_{0}, D_{i j}\left(\Delta_{0}\right)=D_{i j}(\Delta)_{0}
$$

the operation $C_{i j}$ does not change the values of $s_{\nu}$, and the operation $D_{i j}$ replaces $s_{i}$ by $s_{i}+s_{j}$ leaving the other values $s_{\nu}$ unchanged.

We call a function $F(\Delta)$ on the set of symmetric matrices a Tutte polynomial if the following properties hold. 
(A) For every pair of distinct indices $i$ and $j$,

$$
F(\Delta)=F\left(D_{i j}(\Delta)\right)-\Delta_{i j} F\left(C_{i j}(\Delta)\right)
$$

(B) Let $\Delta^{\prime}$ and $\Delta^{\prime \prime}$ be two matrices with indices in $V^{\prime}$ and $V^{\prime \prime}$, and let $\Delta=\Delta^{\prime} \times \Delta^{\prime \prime}$ be a matrix with indices in disjoint union of $V^{\prime}$ and $V^{\prime \prime}, \Delta_{i j}=\Delta_{i j}^{\prime}$, for $i, j \in V^{\prime}, \Delta_{i j}=\Delta_{i j}^{\prime \prime}$ for $i, j \in V^{\prime \prime}, \Delta_{i j}=0$ otherwise. Then

$$
F\left(\Delta^{\prime} \times \Delta^{\prime \prime}\right)=F\left(\Delta^{\prime}\right) F\left(\Delta^{\prime \prime}\right)
$$

Let $T(\Delta)$ be a function of a symmetric matrix which does not depend on the values of $s_{i}$, satisfies $(\mathrm{A})$, and is equal to 1 for a $1 \times 1$ matrix $\Delta$ and to zero for any diagonal matrix of size greater than 1 . Then $T(\Delta)$ coincides with the tree number $T(G)$ of a graph $G$ in case $\Delta=\Delta(G)$.

Let $\Delta$ satisfy (1) and (23). Consider the set of periodic trajectories of the AA model with the matrix $\Delta$ and the loading rate $v_{\nu}=s_{\nu}$, for all $\nu$. The same arguments as in Appendix $\mathrm{F}$ show that the volume $X\left(\Delta ; V_{1}, \ldots, V_{m} ; \mathbf{i}\right)$ occupied by all recurrent configurations generating periodic trajectories with an ordered set of avalanches covering subsets $V_{1}, \ldots, V_{m}$ of $V$ starting at sites $i_{\nu} \in V_{\nu}$ satisfies

$$
X\left(\Delta ; V_{1}, \ldots, V_{m} ; \mathbf{i}\right)=X\left(C_{i j}(\Delta) ; V_{1}, \ldots, V_{m} ; \mathbf{i}\right)-\frac{\Delta_{i j} s_{i}}{s_{i}+s_{j}} X\left(D_{i j}(\Delta) ; V_{1}, \ldots, \hat{V}_{\nu}, \ldots, V_{m} ; \mathbf{i}\right)
$$

when $i=i_{\nu}, j \in V_{\nu}, j \neq i, \hat{V}_{\nu}=V_{\nu} \backslash\{j\}$, and

$$
X\left(\Delta ; V_{1}, \ldots, V_{m} ; \mathbf{i}\right)=s_{1} \cdots s_{m}
$$

when $V_{\nu}=\left\{i_{\nu}\right\}$, for all $\nu$. Hence

$$
X\left(\Delta ; V_{1}, \ldots, V_{m} ; \mathbf{i}\right)=s_{i_{1}} T\left(\Delta_{1}\right) \ldots s_{i_{m}} T\left(\Delta_{m}\right)
$$

where $\Delta_{\nu}$ is the minor of $\Delta$ with indices in $V_{\nu}$. 
Let $f_{m}(\Delta)$ be the volume of all periodic trajectories with $m$ avalanches. Due to (49),

$$
f_{m}(\Delta)=\sum_{V_{1}, \ldots, V_{m}} S\left(V_{1}\right) T\left(\Delta_{1}\right) \cdots S\left(V_{m}\right) T\left(\Delta_{m}\right) .
$$

Here the sum is taken over all partitions of $V$ into $m$ subsets, $S\left(V_{\nu}\right)=\sum_{i \in V_{\nu}} s_{i}$.

Let $F(z)(\Delta)=\sum_{m} f_{m}(\Delta) z^{m}$. Due to (50), $F(z)(\Delta)$ satisfies (45) and (46), i.e. $F(z)$ is a Tutte polynomial. In particular, the total volume of all periodic trajectories is equal to $F(1)=\operatorname{det}(\Delta)$. Hence $\operatorname{det}(\Delta)$ satisfies (45). This implies, in particular, the following identity.

$$
F(z)(\Delta)=\operatorname{det}\left(\Delta_{0}+z \mathbf{s}\right)
$$

where $\mathbf{s}$ is a diagonal matrix with $\mathbf{s}_{i i}=s_{i}$.

Computing linear terms in $S$ in the expression for $F(1)$, we have the Matrix-Tree Theorem

$$
T(\Delta)=\operatorname{det}\left(\Delta_{0}(i)\right) \text {, for every } i \in V(G) .
$$

Here $\Delta_{0}(i)$ is the matrix $\Delta_{0}$ with the $i$-th row and column removed.

Finally, the mean number of avalanches per unit time in a randomly chosen periodic trajectory is equal to

$$
F^{\prime}(1) / \operatorname{det}(\Delta)=\sum_{i}\left(\Delta^{-1}\right)_{i i} \sum_{j} \Delta_{i j}
$$

7. Conclusions. We introduce a class of deterministic lattice models of failure with continuous phase variables, Abelian avalanche (AA) models, with Abelian properties similar to those of the discrete, stochastic Abelian sandpile (ASP) models. We investigate analytically the dynamics, distributions of avalanches and the structure of the phase space of AA models. Depending on the loading rate vector, the steady state dynamics of the AA model can be periodic or quasiperiodic. However, periodic trajectories can contain sequences of avalanches with non-trivial time-space-size distributions. We call this phenomenon "periodic chaos". We show, in particular, that the distribution of avalanches for an ASP model is identical to the distribution of avalanches for an AA model with the same 
redistribution matrix and loading rate vector, after averaging over all periodic trajectories. We present a proof of Dhar's conjecture on the description of the set of recurrent configurations of an Abelian model in terms of forbidden subconfigurations. Recurrent combinatorial formulas for the distributions of avalanches are given, in terms of operations on matrices corresponding to deletion and contraction operations in graph theory. Corresponding combinatorial expressions are known in graph theory as Tutte polynomials. Several identities for these combinatorial expressions, in terms of determinants of various matrices, are derived.

\section{APPENDIX}

A. Proof of (7). Let $\mathbf{n}=\left\{n_{i}, i \in V\right\}$ where $n_{i}$ is the number of breaks of a site $i$ during a time interval $t$, starting from a stable configuration $\mathbf{h}$, and let $\mathbf{h}^{\prime}$ be the stable configuration after these breaks. Then $\mathbf{h}^{\prime}=\mathbf{h}+\mathbf{v} t-\Delta^{\prime} \mathbf{n}$ where $\mathbf{v}$ is the loading rate vector in a deterministic model or its mean value during a time interval $t$ in a stochastic model.

As both configurations $\mathbf{h}$ and $\mathbf{h}^{\prime}$ belong to $\mathcal{S}$, the distance between $\mathbf{h}^{\prime \prime}$ and $\mathbf{h}$ remains bounded, hence

$$
\Delta^{\prime} \mathbf{n} / t=\mathbf{v}+\left(\mathbf{h}-\mathbf{h}^{\prime}\right) / t \rightarrow \mathbf{v} \text { as } t \rightarrow \infty
$$

B. Proof of (8). We want to prove that any two avalanches starting at a point in $\mathbf{R}_{+}^{V}$ terminate at the same stable point. In this case the two avalanches automatically contain equal number of breaks for every site. Let $\mathbf{i}=\left(i_{1}, \ldots, i_{l}\right)$ be an avalanche of minimum size $l$ such that there exists another avalanche $\mathbf{j}=\left(j_{1}, \ldots, j_{m}\right)$ starting at the same point $q$ with a different end. Let $q^{\prime} \neq q^{\prime \prime}$ be the ends of $\mathbf{i}$ and $\mathbf{j}$. Then $i_{1} \neq j_{1}$, otherwise $l$ is not a minimum. We want to show that $p=q-\delta_{i_{1}}-\delta_{j_{1}}$ belongs to $\mathbf{R}_{+}^{V}$. As $q-\delta_{i_{1}} \in \mathbf{R}_{+}^{V}$, only the coordinate $j_{1}$ of $p$ may be negative. The same argument with $i_{1}$ and $j_{1}$ interchange shows that only the coordinate $i_{1}$ of $p$ may be negative. As $i_{1} \neq j_{1}, p \in \mathbf{R}_{+}^{V}$. In particular, $l>1$ and $m>1$. Let $\mathbf{k}$ be an avalanche initiated at $p$. An avalanche $\mathbf{i}^{\prime}=\left(i_{2}, \ldots, i_{l}\right)$ initiated at $q-\delta_{i_{1}}$ has size $l-1$, hence an avalanche $\left(j_{1}, \mathbf{k}\right)$ initiated at $q-\delta_{i_{1}}$ has the same end $q^{\prime}$ 
and size $l-1$ as $\mathbf{i}^{\prime}$. Next, its end coincides with the end of the avalanche $\left(i_{1}, \mathbf{k}\right)$ initiated at $q-\delta_{j_{1}}$, As the size of this last avalanche is $l-1$, the avalanche $\left(j_{2}, \ldots, j_{m}\right)$ initiated at $q-\delta_{j_{1}}$ has the same end $q^{\prime}$, i.e. $q^{\prime}=q^{\prime \prime}$ in contradiction with our hypothesis.

If an avalanche contains a loading vector $\mathbf{v}$ with $v_{i} \geq 0$, for all $i$ at some site, the same argument shows that an avalanche with the vector $v$ displaced one site towards the starting point always belongs to $\mathbf{R}_{+}^{V}$. This proves commutativity of loading-avalanche operators.

C. Proof of (11). Let $\mathbf{h}$ be a configuration in $\mathcal{S}_{\mathbf{u}}$, and let $\mathbf{h}^{\prime}$ be any configuration with large enough components equivalent to $\mathbf{h}$. We want to show that

$$
\mathcal{A} \mathbf{h}=\mathcal{A} \mathbf{h}^{\prime}
$$

This shows that avalanches started at any two equivalent configurations in $\mathcal{S}_{\mathbf{u}}$ terminate at the same stable configuration, hence $\mathcal{A}\left(\mathcal{S}_{\mathbf{u}}\right)$ is a fundamental domain for $\mathcal{L}$.

To prove (54), we note that condition of (11) implies that $\mathbf{h}-\mathcal{A} \mathbf{h}^{\prime}=\sum n_{i} \delta_{i}$ where all $n_{i}$ are non-negative. Because the components of $\mathbf{h}$ and $\mathcal{A} \mathbf{h}^{\prime}$ and the values of $n_{i}$ are bounded, we can suppose that any sequence of breaks, with at most $n_{i}$ breaks at a site $i$, applied to $\mathbf{h}^{\prime \prime}=\mathbf{h}^{\prime}+\mathbf{h}-\mathcal{A} \mathbf{h}^{\prime}$ is contained in $\mathbf{R}_{+}^{V}$ (this is the exact meaning of "large enough" components of $\mathbf{h}^{\prime}$ ). Hence there exists an avalanche started at $\mathbf{h}^{\prime \prime}$ passing through $\mathbf{h}^{\prime}$. Due to the Abelian property (8), this yields $\mathcal{A} \mathbf{h}^{\prime \prime}=\mathcal{A} \mathbf{h}^{\prime}$. At the same time, an avalanche from $\mathbf{h}^{\prime}$ to $\mathcal{A} \mathbf{h}^{\prime}$ shifted by $\mathbf{h}-\mathcal{A} \mathbf{h}^{\prime}$ (due to the condition of (11), all components of this vector are non-negative) connects $\mathbf{h}^{\prime \prime}$ with $\mathbf{h}$. Due to the Abelian property (8), this yields $\mathcal{A} \mathbf{h}^{\prime \prime}=\mathcal{A}$. Hence, $\mathcal{A} \mathbf{h}^{\prime}=\mathcal{A} \mathbf{h}$, q.e.d.

D. Proof of (19). We call a (stable or unstable) configuration $\mathbf{h}$ reachable if there exists an avalanche passing through $\mathbf{h}$ and starting at a configuration with arbitrarily large components.

Let us show first that configurations in $\mathcal{V}_{\mathbf{n}}$ are not reachable. If some of the configurations in $\mathcal{V}_{\mathbf{n}}$ are reachable, then all configurations in $\mathcal{V}_{\mathbf{n}}$ close to $\mathbf{Q}_{\mathbf{n}}$ are reachable. There exists a configuration $\mathbf{h}$ in $\mathcal{R}$ arbitrarily close to $\mathbf{Q}$. The configuration $\mathbf{h}_{\mathbf{n}}=\mathbf{h}-\Delta^{\prime} \mathbf{n}$ is 
equivalent to $\mathbf{h}$, belongs to $\mathcal{V}_{\mathbf{n}}$ and is close to $\mathbf{Q}_{\mathbf{n}}$. Any avalanche starting at a configuration with large enough components that passes through $\mathbf{h}_{\mathbf{n}}$ should terminate at $\mathbf{h} \in \mathcal{R}$. But this is possible only if all components of $\mathbf{n}$ are non-positive. Hence $\mathcal{R} \subset \mathcal{S} \backslash \cup^{\prime} \mathcal{V}_{n}$. It is easy to show that for any two equivalent configurations in $\mathcal{S}$ at least one belongs to $\cup^{\prime} \mathcal{V}_{n}$. As $\mathcal{R}$ is a fundamental domain for the lattice $\mathcal{L}$, this yields $\mathcal{R}=\mathcal{S} \backslash \cup^{\prime} \mathcal{V}_{n}$

E. Proof of (21). Due to (19),

$$
\mathcal{R} \subset \mathcal{S} \backslash \bigcup_{X \subset V} \mathcal{F}_{X}
$$

Hence it is enough to show that the volume of the right side in (55) is $\operatorname{det}(\Delta)$ if $(22)$ holds. We have

$$
\operatorname{Vol}\left(\mathcal{S} \backslash \bigcup_{X \subset V} \mathcal{F}_{X}\right)=\prod_{i \in V} \Delta_{i i}+\sum_{l \geq 1}(-1)^{l} \sum_{X_{1}, \ldots, X_{l}} \operatorname{Vol}\left(\mathcal{F}_{X_{1}} \cap \ldots \cap \mathcal{F}_{X_{l}}\right)
$$

Here the sum is taken over all unordered collections $X_{1}, \ldots, X_{l}$ of distinct nonempty subsets of $V$.

If (22) holds then, for any two subsets $X^{\prime}$ and $X^{\prime \prime}$ of $V$,

$$
\mathcal{F}_{X^{\prime}} \cap \mathcal{F}_{X^{\prime \prime}} \subset \mathcal{F}_{X^{\prime} \cup X^{\prime \prime}}
$$

This implies that only terms with

$$
X_{1} \subset \ldots \subset X_{l}
$$

can be left in (56).

To show this, let $\prec$ be any ordering of subsets of $V$ such that $X^{\prime} \prec X^{\prime \prime}$ when $\left|X^{\prime}\right|<$ $\left|X^{\prime \prime}\right|$ and the sets of equal size are arbitrarily ordered. Then $X_{j}$ in (56) can be arranged in increasing order

$$
X_{1} \prec \ldots \prec X_{l} \text {. }
$$

Let $X_{j}$ be the first term in (59) such that $X_{j} \not \subset X_{j+1}$. Then $X_{j+1} \prec X_{j} \cup X_{j+1}$. If the sequence (59) contains $X_{j} \cup X_{j+1}$, we remove it from the sequence, otherwise we add it to 
the sequence. Due to (57) this operation does not change the value of the corresponding term in (56) but does change its sign. Hence all terms but (58) annihilate in (56).

If (22) holds then

$$
\operatorname{Vol}\left(\mathcal{F}_{X_{1}} \cap \ldots \cap \mathcal{F}_{X_{l}}\right)=\prod_{j \notin X_{l}} \Delta_{j j} \prod_{i=1}^{l} \prod_{j \in X_{i} \backslash X_{i-1}}\left(-\sum_{\nu \in X_{i}, \nu \neq j} \Delta_{\nu j}\right),
$$

for $X_{1} \subset \ldots \subset X_{l}$, appears in (56) with the sign $(-1)^{l}$. If we add an empty set $\emptyset$ as $X_{0}$ to every sequence (58) and define $\mathcal{F}_{\emptyset}=\mathcal{S}$, then (56) can be rewritten as

$$
\sum_{l \geq 0}(-1)^{|V|-\left|X_{l}\right|} \sum_{X_{0} \subset X_{1} \subset \ldots \subset X_{l}} \prod_{j \notin X_{l}} \Delta_{j j} \prod_{i=1}^{l} \prod_{j \in X_{i} \backslash X_{i-1}} \sum_{\nu \in X_{i}, \nu \neq j} \Delta_{\nu j} .
$$

We claim that this is equal to $\operatorname{det}(\Delta)$, for any matrix $\Delta$.

Expanding all the sums and products in (61) we can rewrite it in the following way:

$$
\sum_{\varphi} \varepsilon(\varphi) \prod_{j \in V} \Delta_{\varphi(j), j},
$$

where $\varphi$ runs over all maps from $V$ to itself, and the coefficient $\varepsilon(\varphi)$ is defined as follows. Let $V^{\varphi}=\{j \in V: \varphi(j)=j\}$ be the set of fixed points of $\varphi$. Then

$$
\varepsilon(\varphi)=(-1)^{\left|V \backslash V^{\varphi}\right|} \sum_{\mathbf{X}}(-1)^{l(\mathbf{X})},
$$

where the sum is taken over all $\varphi$-invariant flags

$$
\mathbf{X}=\left\{\emptyset=X_{0} \subset X_{1} \subset \ldots \subset X_{l}=V \backslash V^{\varphi}\right\}
$$

and $l(\mathbf{X})=l$ is the length of $\mathbf{X}$. Here the $\varphi$-invariance of $\mathbf{X}$ means that $\varphi\left(X_{i}\right) \subseteq X_{i}$, for all $i$.

If $\varphi$ is a permutation of $V$ then every $\varphi$-invariant set in $V \backslash V^{\varphi}$ is identified by a subset of the set $W$ of cycles of $\varphi$ with length greater than 1 .

All subsets of $W$ can be identified with vertices of an $|W|$-dimensional cube if we set an $i$-th component of a vertex equal to 1 when $i$ belongs to a subset and 0 otherwise. All flags 
of subsets of $W$ constitute a simplicial complex (with the length of a flag as the dimension of a simplex) which is a simplicial subdivision of this cube. The flags starting with $\emptyset$ and ending with $W$ constitute an open $|W|$-dimensional cube. Its Euler characteristics $\chi=(-1)^{|W|}$.

Hence $\varepsilon(\varphi)$ is equal to $(-1)^{|V|-k}$ where $k$ is the number of all cycles of $\varphi$ which is the usual sign of a permutation.

In case $\varphi$ is not a permutation, there exist two $\varphi$-invariant subsets $A \supset B$ in $V \backslash V^{\varphi}$ such that $|A|=|B|+1, \varphi(A)=B$ and $B$ does not contain non-trivial $\varphi$-invariant subsets. For every flag $\mathbf{X}$, we find $i$ such that $X_{i+1} \supseteq A, X_{i} \not \supset A$. Then $X_{i} \subseteq Y=\left(X_{i+1} \backslash A\right) \cup B$. If $X_{i}=Y$ we remove it from the flag, otherwise we add $Y$ to the flag between $X_{i}$ and $X_{i+1}$. This operation defines a sign-changing isomorphism of the set of terms in the sum (63). Hence $\varepsilon(\varphi)=0$.

F. Proof of (31). First, it is obvious that (31) is positive only when all subgraphs $G_{\nu}$ are connected. Also, avalanches covering a non-connected graph have zero measure, because to start such an avalanche at least one site at every connected component have to be at the threshold level.

Configurations generating trajectories with a sequence of single breaks $i_{1}, \ldots, i_{N}$ occupy a simplex

$$
0<t_{1}=\frac{\Delta_{i_{1} i_{1}}-h_{i_{1}}}{s_{i_{1}}}<\ldots<t_{N}=\frac{\Delta_{i_{1} i_{N}}+\ldots+\Delta_{i_{N} i_{N}}-h_{i_{N}}}{s_{i_{N}}} \leq 1
$$

where $t_{\nu}$ are the time moments of breaks at $i_{\nu}$. Its volume is equal to $s_{i_{1}} \cdots s_{i_{N}} / N$ !. Hence (31) is true in this case.

For partitions where at least one subgraph has order greater than 1, we proceed by induction on the number of edges of the graph $G$ and suppose that the statement is true for both $G-e$ and $G / e$ where $e$ is any edge of $G$.

Let $G_{1}, \ldots, G_{m}$ be a partition of $G$ into induced connected subgraphs, $\left|G_{\nu}\right|>1$, and $i_{\nu} \in G_{\nu}$ a site starting an avalanche. Then there exists an edge $e$ of $G$ with one end $i_{\nu}$ and 
another end $j \in G_{\nu}$.

Let $\mathbf{h}(\mathbf{t})=\left\{h_{i}(t), i \in V(G)\right\}$, be a periodic trajectory with avalanches $G_{1}, \ldots, G_{m}$ and starting sites $i_{1}, \ldots, i_{m}$, and let $\mathbf{h}=\left\{h_{i}\right\}=\mathbf{h}\left(t_{\nu}\right)$ where $t_{\nu}$ is the time moment of the avalanche $G_{\nu}$. Then $h_{i_{\nu}}=\Delta_{i_{\nu} i_{\nu}}$.

There are two possibilities.

(a) $h_{j}<\Delta_{j j}-1$.

Replacement of $h_{i_{\nu}}(t)$ by $h_{i_{\nu}}(t)-1$ defines a one-to-one correspondence between trajectories for the system on $G$ satisfying (a) and all trajectories for a system on $G-e$, with the same values of $s_{i}$, generating the partition induced from $G$, with the same starting points and time moments of breaks. Due to inductional conjecture, the volume of the configurations generating trajectories satisfying (a) is equal to

$$
s_{i_{1}} T\left(G_{1}\right) \cdots s_{i_{\nu}} T\left(G_{\nu}-e\right) \cdots s_{i_{m}} T\left(G_{m}\right) / m !
$$

(b) $\Delta_{j j}>h_{j} \geq \Delta_{j j}-1$.

We can identify the trajectory $\mathbf{h}(t)$ with a periodic trajectory for a system on $G / e$, with the site $j$ removed and all the edges adjacent to it connected to the site $i_{\nu}$, passing at the time moment $t_{\nu}$ through the configuration obtained from $\mathbf{h}$ replacing $h_{i_{\nu}}=\Delta_{i_{\nu} i_{\nu}}$ by the threshold value $\Delta_{i_{\nu} i_{\nu}}+\Delta_{j j}+2 \Delta_{i_{\nu} j}-s_{j}$ for $G / e$. This trajectory generates the partition of $G / e$ induced from $G$, with the same starting points and time moments of breaks. The correspondence represent the set of trajectories for $G$ satisfying (b) as a prism of height 1 over the set of trajectories for $G / e$ generating partition induced from $G$, with the same starting points and time moments of breaks. From the induction conjecture, the volume of the configurations generating trajectories satisfying (b) is equal to

$$
s_{i_{1}} T\left(G_{1}\right) \cdots s_{i_{\nu}} T\left(G_{\nu} / e\right) \cdots s_{i_{m}} T\left(G_{m}\right) / m !
$$

Then, due to (30), the sum of (66) and (67) is equal to (31). This proves our claim.

Acknowledgements. This work was performed when the author was visiting the Department of Geology, Cornell University, under NSF grant \#EAR-91-04624, and the Institute 
for Theoretical Physics, UCSB, under NSF grant \#PHY89-04035. The author thanks D.L.Turcotte, W.I.Newman, L.Billera, A.Zelevinsky, and G.Narkounskaya for discussions and correspondence, and especially W.I.Newman for many suggestions during the preparation of the manuscript.

\section{REFERENCES}

[1] P. Bak, C. Tang and K. Wiesenfeld, Phys. Rev. Lett. 59, 381 (1987); Phys. Rev. A 38, $364(1988)$.

[2] C. Tang and P. Bak, Phys. Rev. Lett. 60, 2347 (1988); J. Stat. Phys. 51, 797 (1988); L.P. Kadanoff, S.R. Nagel, L. Wu and S. Zhou, Phys. Rev. A 39, 6524 (1989); B. Chhabra, M.J. Feigenbaum, L.P. Kadanoff, A.J. Kolan and I. Procaccia, Sandpiles, Avalanches and the Statistical Mechanics of Non-Equilibrium Stationary States, Preprint (1992); T. Hwa and M. Kardar, Phys. Rev. Lett. 62, 1813 (1989); Physica D 38, 198 (1989); Phys. Rev. A 45, 7002 (1992); S.S. Manna, J. Stat. Phys. 59, 509 (1990); Physica A 179, 249 (1991); C.-H. Liu, H.M. Jaeger and S.R. Nagel, Phys. Rev. A 43, 7091 (1991).

[3] D. Dhar, Phys. Rev. Lett. 64, 1613 (1990).

[4] D. Dhar and S.N. Majumdar, J. Phys. A: Math. Gen. 23, 4333 (1990); S.N. Majumdar and D. Dhar, J. Phys. A: Math. Gen. 24, L357 (1991); Physica A 185, 129 (1992); D. Dhar, Physica A 186, 82 (1992); P. Grassberger and S.S. Manna, J. Phys. France 51, 1077 (1990); M. Creutz, Nucl. Phys. B, Proc. Suppl. 20, 758 (1991); Comput. Phys., 5, 198 (1991); E. Goles, Ann. Inst. Henri Poincare - Phys. Theor. 56, 75 (1992); H.F. Chau and K.S. Cheng, Phys. Rev. A 44, 6233 (1991); Phys. Rev. A 46, R2981 (1992).

[5] B. Barriere and D.L. Turcotte, Geophys. Res. Lett. 18, 2011 (1991). 
[6] A. Bjorner, L. Lovasz and P.W. Shor, Europ. J. Combinatorics 12, 283 (1991); A. Bjorner and L. Lovasz, Chip firing games on directed graphs, Preprint (1991).

[7] R. Burridge and L. Knopoff, Bull. Seismol. Soc. Amer. 57, 341 (1967).

[8] J. Carlson and J.S. Langer, Phys. Rev. Lett. 62, 2632 (1989); Phys. Rev. A 40, 6470 (1989); J.M. Carlson, Phys. Rev. A 44, 6226 (1991).

[9] H. Nakanishi, Phys. Rev. A 41, 7086 (1990); Phys. Rev. A 43, 6613 (1991); M. Matsuzaki and H. Takayasu, J. Geophys. Res. 96, 19925 (1991); J. Lomnitz-Adler, L. Knopoff and G. Martinez-Mekler, Phys. Rev. A 45, 2211 (1992).

[10] H.J.S. Feder and J. Feder, Phys. Rev. Lett. 66, 2669 (1991); A. Diaz-Guilera, Phys. Rev. A 45, 8551 (1992).

[11] Z. Olami, H.J.S. Feder and K. Christensen, Phys. Rev. Lett. 68, 1244 (1992); K. Christensen and Z. Olami, Phys. Rev. A 46, 1829 (1992).

[12] Y.-C. Zhang, Phys. Rev. Lett. 63, 470 (1989); L. Pietronero, P. Tartaglia and Y.-C. Zhang, Physica A 173, 22 (1991).

[13] A. Gabrielov, W.I. Newman and L. Knopoff, Lattice models of failure: sensitivity to the local dynamics, Preprint (1992).

[14] W.T. Tutte, Graph Theory, Addison-Wesley, 1984. 